\title{
Breakpoint sequences of an 1;8 translocation in a family with Gilles de la Tourette syndrome
}

\author{
Naomichi Matsumoto ${ }^{1}$, Donna E David ${ }^{2}$, Eric W Johnson ${ }^{2}$, David Konecki ${ }^{2}$, \\ James K Burmester ${ }^{2}$, David H Ledbetter ${ }^{1}$ and James L Weber ${ }^{2}$
}

\begin{abstract}
${ }^{1}$ Department of Human Genetics, The University of Chicago, Chicago, IL; ${ }^{2}$ Center for Medical Genetics, Marshfield Medical Research Foundation, Marshfield, WI, USA
\end{abstract}

\begin{abstract}
Gilles de la Tourette syndrome (GTS) is a common, heritable neurological disorder manifested by chronic motor and vocal tics with childhood onset. Previous extensive linkage analysis failed to identify a GTS gene based on an autosomal dominant pattern of inheritance. Recently, a family was reported with a balanced chromosomal translocation $\mathrm{t}(1 ; 8)(q 21.1 ; q 22.1)$ in family members with GTS or tics. Chromosome 8q22.1 was previously implicated in GTS by both association and linkage results. We therefore cloned and sequenced both translocation breakpoints from this family. The CBFA2T1 gene was identified $11 \mathrm{~kb}$ distal to the 8q22.1 breakpoint. Sequencing of CBFA2TI exons within 37 unrelated GTS patients failed to identify any mutations. However, it is possible that the translocation altered the expression of this gene or another nearby gene. Examination of the breakpoint sequences revealed a duplication of six nucleotides from chromosome 8 but no change in the chromosome 1 sequence. The sequences immediately flanking the breakpoints on the two chromosomes were modestly similar, but the breakpoints did not occur within known interspersed repeats. Our results add to our knowledge of the genetics of GTS and the mechanisms of balanced chromosomal translocations. European Journal of Human Genetics (2000) 8, 875-883.
\end{abstract}

Keywords: Gilles de la Tourette syndrome; chromosomal translocation; positional cloning; chromosome8; CBFA2T1

\section{Introduction}

Gilles de la Tourette Syndrome (GTS) (MIM137580) is characterized by chronic involuntary motor and vocal tics with childhood onset. ${ }^{1}$ GTS can also present with obsessivecompulsive disorder and attention deficit disorder. Strong heritability of GTS has been concluded from both twin and family studies. ${ }^{2,3}$ Concordances for full GTS were $53 \%$ or $56 \%$ for monozygotic (MZ) and 8\% for dizygotic (DZ) twins. If chronic motor tics were included in the diagnoses, concordances would be $77 \%$ or $94 \%$ for MZ and $23 \%$ for DZ twins, respectively. ${ }^{4,5}$ Initially, GTS was predicted to be inherited in an autosomal dominant fashion with incomplete penetrance and variable expression, ${ }^{6-8}$ but extensive linkage analysis failed to identify a gene based on this mode of inheritance. $^{9-11}$ This could be due to uncertain phenotype spec-

Correspondence: James L Weber PhD, Center for Medical Genetics, Marshfield Medical Research Foundation, 1000 North Oak Avenue, Marshfield, WI 54449, USA. Tel: +1715 387 9179; Fax: +1715 389 5757; E-mail: weberj@cmg.mfldclin.edu

Received 17 March 2000; revised 23 June 2000; accepted 7 July 2000 trum, locus heterogeneity, or polygenic inheritance. Recent segregation studies suggested a complex model of inheritance. $^{12,13}$ Furthermore, clinical evidence of genomic imprinting and bilineal transmission have been reported. ${ }^{14,15}$

More recently, results of whole genome sib pair and association studies have been reported. Using 110 affected sib pairs, The Tourette Syndrome Genetic Consortium ${ }^{16}$ reported evidence for linkage on chromosomes $8 p$ and $4 q$. Simonic et $\mathrm{al}^{17}$ reported association of GTS among South African Afrikaners to chromosomes $2,6 p, 8 q, 11 q, 14 q, 20 q$ and $21 q$.

At least five chromosomal abnormalities have been reported in GTS patients. These include a $9 p$ deletion, ${ }^{18}$ 18q22.2 deletion, ${ }^{19} \mathrm{t}(7 ; 18)(\mathrm{q} 22 ; \mathrm{q} 22.3){ }^{20} \mathrm{t}(3 ; 8) \quad$ (p21.3; $\mathrm{q} 24.1),{ }^{21}$ and most recently a $\mathrm{t}(1 ; 8)(\mathrm{q} 21.1 ; \mathrm{q} 22.1) .{ }^{22}$ The last report involved a nuclear family in which the father and six of the seven children shared the balanced $(1 ; 8)$ translocation. Of the six children with the translocation, one had full blown GTS, one displayed modest tics and Asperger's syndrome, two 
had mild tics, and the two youngest were unaffected but were also below the average age of onset.

The site of the chromosome 8q22.1 breakpoint within the $t(1 ; 8)$ family was the same, within confidence intervals, as the $8 q$ association region reported by Simonic et al. ${ }^{17}$ This same region was also implicated by linkage analysis in a large Utah GTS kindred. ${ }^{23}$ We therefore set out to map the 8q22.1 breakpoint within the translocation family. We report here the cloning and sequencing of both the chromosome 1 and 8 breakpoints from this family and the identification of the CBFA2T 1 gene $11 \mathrm{~kb}$ distal of the $8 q$ breakpoint.

\section{Materials and methods \\ Fluorescence in situ hybridization}

FISH using BAC, cosmid and phage DNA was performed on metaphases of the patients with the 1;8translocation and a normal control. Chromosome preparations were made from lymphoblastoid cell lines by conventional methods. Slides were incubated in $2 \times \mathrm{SSC}$ at $37^{\circ} \mathrm{C}$ for $30 \mathrm{~min}$, serially dehydrated in $70 \%, 80 \%$, and $95 \%$ ethanol at room temperature, denatured in $70 \%$ formamide $/ 2 \times$ SSC at $72^{\circ} \mathrm{C}$ for $2 \mathrm{~min}$, then serially dehydrated at $-20^{\circ} \mathrm{C}$ in $70 \%, 80 \%, 90 \%$, and $100 \%$ ethanol. Clone DNAs were labeled with digoxigenin-11-dTUP (Boehringer-Mannheim, Indianapolis, IN, USA) by nick translation, precipitated in ethanol with a $50 \mathrm{X}$ excess of human Cot-1 and herring testis DNA (Gibco-BRL, Gaithersburg, MD, USA), and resuspended to a final concentration of $20 \mathrm{ng} / \mu \mathrm{l}$ in hybridization solution $(50 \%$ formamide, $2 \times$ SSC, $10 \%$ dextran sulfate). Probes were denatured at $76^{\circ} \mathrm{C}$ for $10 \mathrm{~min}$ and preassociated at $37^{\circ} \mathrm{C}$ for $15 \mathrm{~min}$ prior to hybridization. The probe-hybridization mix ( $10 \mu \mathrm{l})$ was applied under a $22 \mathrm{~mm} \times 22 \mathrm{~mm}$ coverslip and slides were incubated in a moist chamber for $16 \mathrm{~h}$ at $37^{\circ} \mathrm{C}$, then washed as previously described. ${ }^{24}$ Probes were detected at $50 \mu \mathrm{l}$ of rhodamine anti-digoxigenin (Boehringer-Mannheim) at $1 \mu \mathrm{g} / \mu \mathrm{l}$. Slides were washed three times in $4 \times$ SSC, $0.1 \%$ Tween -20 at $45^{\circ} \mathrm{C}$ and mounted in antifade solution (Vector, Burlingame, NY, USA) containing DAPI. Analysis was performed using a (Zeiss, Thornwood, NY, USA) Axiophot microscope equipped with filters to detect DAPI and rhodamine separately, as well as a triple band pass filter (Chroma Technology Corporation, Brattleboro, VT, USA) to detect signals simultaneously. Images were collected and merged using a cooled CCD camera (KAF 1400, Photometrics, Tucson, AZ, USA) and Quips M-FISH software (Vysis Inc, Downers Grove, IL, USA).

\section{Isolation of BACs, cosmids, phages and plasmids} Human BAC libraries (Research Genetics, Huntsville, AL, USA; Genome Systems, St Louis, MO, USA) were screened using PCR. The size of BACs spanning the breakpoints was determined by pulsed-field gel electrophoresis analysis as described previously. ${ }^{25}$
Cosmid and phage sublibraries were prepared from BACs spanning the breakpoint. Purified BAC DNA was isolated using Qiagen Midi-Prep columns (Chatsworth, CA, USA) and digested partially with Sau3AI. The SuperCosl cosmid vector was prepared according to manufacturer's instructions (Stratagene, La Jolla, CA, USA) and ligated to the digested BAC DNA overnight using T4 DNA ligase. Lambda FIXII/X hol vector (Stratagene) after partial filling-in was also ligated to BAC DNA. Each ligation reaction was packaged using the Gigapack III gold extract (Stratagene) and transfected into XL1-Blue MR host cells (Stratagene) for cosmid or XLI-Blue MRA (P2) host cells (Stratagene) for phage, respectively.

Plasmid subclones for sample sequencing were also prepared from BAC DNA. BAC DNA was isolated using Qiagen Midi-Prep columns and sheared using an ultrasound sonicator. Fragments of 1-2 kb length were cut from an agarose gel, extracted with GenElute ${ }^{T M}$ agarose spin columns (Supelco, Bellefonte, PA, USA), and end-polished with mung bean nuclease. Fragments were ligated into EcoRV-digested pBluescript SK(-) using T4 DNA ligase. Epicurian Coli SoloPack ${ }^{T M}$ supercompetent cells (Stratagene) were used for transformation.

\section{Construction of cosmid and phage contig}

Cosmid DNAs were extracted by an automatic nucleic acid isolation system (AutoGen 740, Integrated Separation Systems, Natick, MA, USA), and phage DNAs were isolated using a Qiagen Lambda mini kit. A total of 24 cosmid clones and 50 phage clones were randomly chosen and used for construction of a contig covering the breakpoint. The contig was constructed by means of STS content mapping using PCR. New STSs were generated from clone end sequences and CBFA 2T 1 exon-specific primers (Gen Bank accession numbers AF018270-AF018282).

\section{Isolation of cosmids spanning the translocation breakpoint}

High molecular weight genomic DNA was prepared from the GTS patient's lymphoblastoid cell line, partially digested with Sau3AI, and ligated to SuperCos1 vector. The ligated products were packaged using the Gigapack III gold extract, and transfected into XL1-Blue MR host cells (Stratagene). A 2.5-genome fold library was plated on $130 \mathrm{~mm}$ dishes, transferred to nylon membranes, and hybridized with two probes flanking the 8q22.1 breakpoint. These probes were PCR products prepared using the following two pairs of primers: $41781 \mathrm{F3}$ (5'-TTT CAC TCTACC TCT GAC AG3')/WI-13899-W 1 (5'-GCATAA GTA AAC CAG CCT TG-3') and 4.7 kb-W 1 (5'-TCT TCC CAA TTA CTAACTAG-3')/cos9T3-W7 (5'-TCATGA GGCTTAAAG GAG GG-3'). Primers 41781F3/ WI-13899-W1 turned out to be $9.1 \mathrm{~kb}$ telomeric to the breakpoint, and primers $4.7 \mathrm{~kb}-\mathrm{W} 1 / \cos 9 \mathrm{~T} 3-\mathrm{W} 7$ were $8.0 \mathrm{~kb}$ centromeric to the breakpoint. Hybridization was carried out in ExpressHyb ${ }^{\mathrm{TM}}$ Hybridization Solution (Clontech, Palo 
Alto, CA, USA) according to the manufacturer's protocol. Blots were washed in $0.1 \times \mathrm{SSC} / 0.1 \times \mathrm{SDS}$ at $50^{\circ} \mathrm{C}$.

\section{DNA sequencing}

BAC, cosmid, and plasmid DNAs were extracted with the AutoGen 740 and purified with Microcon 100 spin columns (Amicon Inc, Beverly, MA, USA). Phage DNA was extracted using a Qiagen lambda mini kit. PCR products were purified using the QIAquick PCR purification kit (Qiagen). Sequencing reactions were carried out with the $A B I$ Prism $^{\mathrm{TM}}$ BigDye Terminator Cycle Sequencing Ready Reaction Kit (PE Applied Biosystems Inc, Foster, CA, USA) using $500 \mathrm{ng}$ of BAC, cosmid, phage, or plasmid DNA or 90 ng of DNA from PCR product as template and $40 \mathrm{pmol}$ (BAC, cosmid, and phage) or $4 \mathrm{pmol}$ (plasmid and PCR product) primer. Products were analyzed on an ABI 377 automated sequencer. Cycle sequencing was performed for 50 cycles (BAC, cosmid, and phage) or 25 cycles (plasmid and PCR) at $96^{\circ} \mathrm{C}$ for $10 \mathrm{~s}, 50^{\circ} \mathrm{C}$ for $5 \mathrm{~s}$, and $60^{\circ} \mathrm{C}$ for $4 \mathrm{~min}$. For complete sequencing of cosmids spanning the breakpoints, a primer walking strategy was adopted. For clone end-sequencing, T7 and SP6 primers for BAC, or T7 and T3 for cosmid, phage, and plasmid were used.

\section{Computational analysis of sequences}

Primer3 (http://www-genome.wi.mit.edu/cgi-bin/primer/ primer3.cgi) was used for generating new STSs from the sequences obtained from clone ends after excluding repetitive DNA sequences such as Alu or L1 with BLASTN (http: // www.ncbi.nlm.nih.gov / cgi-bin / BLAST / nphnewblast? form $=0$ ). For analysis of entire cosmid sequences, RepeatMasker (http://ftp.genome.washington.edu/cgi-bin/ RepeatMasker) was first used for elimination of the repetitive sequences. Then the exon prediction programs GENSCAN (http://ccr-081.mit.edu/GENSCANMIT.html), FGENE (http:/ /genomic.sanger.ac.uk/gf/gf.shtml), and GRAIL2 (http:/ /dmg.nott.ac.uk/molbio/replaced1198/INTRON.HTM) were used to detect potential exons. BLASTN searches were done in GenBank + EMBL + DDBj sequence databases along with dbEST. BLAST2 analysis (http://www.ncbi.nlm.nih.gov/gorf/ b12.html) was performed for comparison of sequences of two cosmids spanning 1q21.1 and 8q22.1 breakpoints.

\section{Results \\ Isolation of BACs spanning the 8q22.1 breakpoint}

To identify BACs spanning the chromosome 8 translocation breakpoint in the GTS family, we isolated at least one BAC clone encompassing each of 10 short tandem repeat polymorphisms (STRPs) near 8q22.1: ATA19G07 (D8S1119), Mfd185A (D8S167), Mfd45A (D8S88), AFM 165yb10 (D8S271), AFM179yf6 (D8S273), AFM287wa5 (D8S1800), AFM 165xh4 (D8S270), GATA28F12, GAAT1A4, and GATA26A08 (D8S1131) (www.marshmed.org/genetics). FISH was then performed by hybridizing DNA from each of these BACs to metaphase chromosome preparations from the GTS patient. Six BACs encompassing ATA19G07, Mfd185A, Mfd45A, AFM 165yb10, AFM179yf6, and AFM287wa5 were mapped proximal to the breakpoint; three BACs encompassing GATA28F12, GAAT1A4, and GATA26A08 were distal, and two BACs (RG302N1 and RG127D17) encompassing AFM165xh4 spanned the 8q22.1 breakpoint (Figurela). These two BACs were also shown to span the chromosome8 breakpoint in the father and the child with Asperger's syndrome. The DNA inserts of RG302N1 and RG127D17 were $140 \mathrm{~kb}$ and $120 \mathrm{~kb}$, respectively.

\section{Identification of ESTs within the chromosome8 breakpoint BACs}

ESTs which map close to AFM 165xh4 were identified using the NCBI GeneMap'99 resource (www.ncbi.nlm.nih.gov/ genemap/). PCR was used to confirm the presence of the CBFA2T 1 gene (also known as MTG8) and WI-13899 (Unigene Hs.144995) on the two BACs spanning the chromosome 8 breakpoint. The CBFA2T1 gene encodes a putative transcription factor and is often joined to the AML1 gene on chromosome 21 in acute myeloid leukemias. ${ }^{26,27}$ The genomic structure of CBFA2T1 has previously been characterized. ${ }^{28}$ The gene has a total of 13 exons distributed over $87 \mathrm{~kb}$ of genomic DNA with transcription orientation from telomere to centromere. ${ }^{26}$ We examined which portions of this gene were present on the two breakpoint BACs by PCR using primers specific for each exon (GenBank accession nos AF018270-AF018282). RG302N1 contained CBFA2T 1 exonsla to 11, and RG127D17 had exons2 to 11 . We also characterized one WI-13899 cDNA clone, IMAGE 37404. The $1.6 \mathrm{~kb}$ long sequence of IMAGE 37404 did not show any long open reading frames (ORFs). This EST al so did not have any introns within the genomic sequence of BAC RG127D17 (see below). Sample sequencing from RG127D17 failed to identify any additional genes. A total of 96 shotgun plasmid clones was sequenced at both ends, and a BLASTN search was performed, but no genes other than CBFA2T 1 were found.

\section{Construction of a cosmid and phage contig for BAC RG127D17}

A complete cosmid and phage contig for BAC clone RG127D17 was constructed by means of STS content mapping using PCR (Figure 2). Besides WI-13899-3', and CBFA2T 1 exons, 2, 5, 6, 8, 10 and 11, six new STSs, RG127D17SP6, RG127D17T7, P18T3, P24T3, cos9T3, and cos4T7, were developed from end sequences of cosmid and phage subclones. FISH mapping revealed that cosmid 9 and phages 18 , 21 and 24 were located proximal to the translocation breakpoint; cosmid 4 and phages 1, 3, 15, 30 and 36 were distal; and cosmid 10 spanned the breakpoint.

\section{Characterization of the cosmid 10 sequence}

The complete 32884 bp sequence of cosmid 10 was determined (GenBank Accession AF198490) and was characterized by computational methods (Figure3a). Human endogenous 

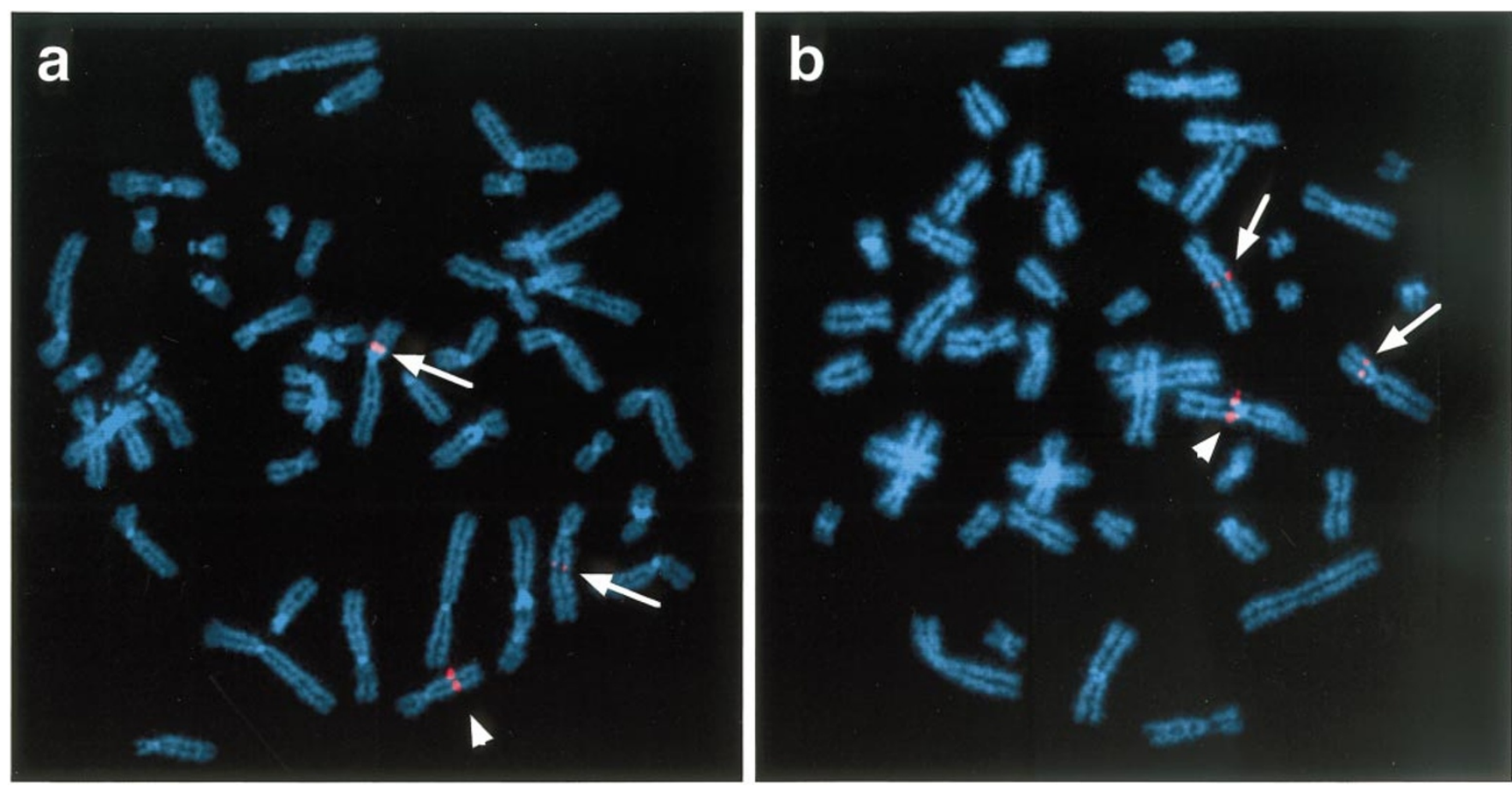

Figure 1 FISH analysis of BACs spanning the breakpoint on the GTS patient. Arrowheads show normal chromosome 8 or 1 , arrows indicate derivative chromosomes. The probes are a RG127D17 spanning the 8q22.1 breakpoint, and b GS156E10 spanning the 1q21.1 breakpoint.

retroviral element $\mathrm{H}$ (HERVH) was identified by RepeatMasker. Exon prediction programs indicated several potential exons, but none of them could be confirmed by BLASTN searches to dbEST except for the CBFA2T1 exon 11. The whole cosmid sequence after masking repetitive sequences with RepeatMasker was used for BLASTN search to dbEST. Hits on two Unigene clusters, Hs. 90858 and Hs. 144995 were obtained. Hs. 90858 and Hs. 14495 are located $8.7 \mathrm{~kb}$ and $7.2 \mathrm{~kb}$ distal, respectively, from the breakpoint (see below). A total of 5IMAGE clones (clones IMAGE 783857, IMAGE 41781, IMAGE 25023 and IMAGE 1692252 from Hs. 90858 and clone IMAGE 37404 from Hs. 144995) were sequenced. These sequences showed no long ORFs, and were identical to the corresponding genomic sequences from cosmid 10 (data not shown). Furthermore, we did not find any expression in fetal tissues by northern analysis using PCR probes from these two Unigene clusters (data not shown). Thus we could not identify any genes spanning the $8 q 22.1$ breakpoint. The last CBFA2T1 exon (exon 11) was $11 \mathrm{~kb}$ distal to the breakpoint.

\section{Cloning of the cosmid containing the 8q22.1 junction fragment}

A cosmid library was constructed from genomic DNA of the GTS patient and screened with two PCR probes, 41781F3/WI13899-W1 and 4.7 kb-W1/cos9T3-W7 (see Methods). One cosmid clone, cos1GTS, was identified which contained the
4.7 kb-W1/cos9T3-W7 probe. Partial sequence analysis of cos1GTS using the same walking primers used for cosmid 10 sequencing revealed that cos1GTS contained some sequence completely different from sequences of cosmid 10 and the cosmid vector. PCR primers were designed using this novel sequence, ch1-W1-F(5'-GCG TTC ATG AAT GAG GTC CC3')/ch1-W1-R(5'-CTG TIT GAA AAT GCC CAAAA-3'), and were used to screen a panel of monochromosomal hybrid DNAs. This screen was positive only for chromosomel DNA (data not shown). Furthermore, FISH analysis of the cos1GTS on normal controls showed signals both at 1q21.1 and 8q22.1 (data not shown).

\section{Identification and characterization of BAC and cosmid clones spanning the 1q21.1 breakpoint}

A total of three BAC cones, GS68016, GS141F1, and GS156E10 were isolated by PCR screening of a normal human BAC library with chromosomel primers from the cos1GTS (ch 1-W1-F/ch1-W1-R). FISH analysis of these three clones on the patient's metaphases showed that all clones spanned the 1q21.1 breakpoint (Figure1b). The insert DNAs of GS68016, GS141F1, and GS156E10 were 40, 110, and $130 \mathrm{~kb}$, respectively. A cosmid sublibrary was constructed from GS156E10 DNA. From 24 randomly chosen cosmid clones, one (1cos4) was identified by PCR primers using primers ch1-W1-F/ch1-W1-R and confirmed to span the 
breakpoint by FISH. Note that 1cos4 is from normal chromosome 1 and is different from chromosome 8 cosmid 4 shown in Figure2.

Cosmid 1cos4 was sequenced completely by a primer walking strategy (GenBank Accession AF198494). Partial sequence from cosiGTS was also added to the 1cos4 sequence. A 36972 bp sequence covering the 1q21.1 breakpoint was thereby obtained and characterized (Figure $3 b$ ). A sequence similar to the KIAA0454 gene (which also maps to chromosome1) was found $21 \mathrm{~kb}$ proximal to the $1 \mathrm{q} 21.1$ breakpoint. This homologue was identified by FGENE and GENSCAN exon-finding programs but not by Grail 2. The KIAA0454 gene was originally isolated from a size-fractionated human brain cDNA library. ${ }^{29}$ The sequence from 1 cos4 of $766 \mathrm{bp}$ of connected exons showed $97 \%$ identity with KIAA0454 at the nucleotidelevel, and its assumed translation product showed $97 \%$ identity with KIAA0454 over 237 amino acids. BLASTN search of this 766 bp sequence to dbEST failed to indentify any perfect match ESTs. Furthermore, expression of the homologue was not confirmed in fetal brain cDNAs by RT-PCR analysis, even though KIAA0454 expression was easily detected (data not shown). This homologue may therefore be an unprocessed pseudogene of KIAA0454.

\section{Sequence comparison of the breakpoints of $\operatorname{der}(1)$ and der(8) chromosomes}

Primers cos10T7-W7 (5'-GTTTCC TTC TAA GCA ACA CA-3') at 8q22.1 and $\operatorname{der}(1)$ R (5'-GAG GTAACT TAA CAC TIT CAG$\left.3^{\prime}\right)$ at 1q21.1 were used for PCR amplification of the der(1) breakpoint. PCR product was obtained and sequenced from the GTS patient. As expected, no product could be amplified from a normal control. The der(8) breakpoint sequence was obtained from cosiGTS as described above. Sequences of normal chromosome 1, 8, der(1), and $\operatorname{der}(8)$ are aligned in Figure 4a. A six nucleotide sequence, 5'-CTTCAC-3', from chromosome8 immediately flanking the breakpoints was duplicated during the translocation process (underlined in Figure 4a). Breakpoint sequences from the father in the translocation family, the GTS patient, and the child with Asperger's syndrome were also compared by PCR direct sequencing. Two sets of primers, cos10T7-W7/der(1)R for the der(1) breakpoint, and ch1F (5'-TGT CAA TAT GCA ATT AAA

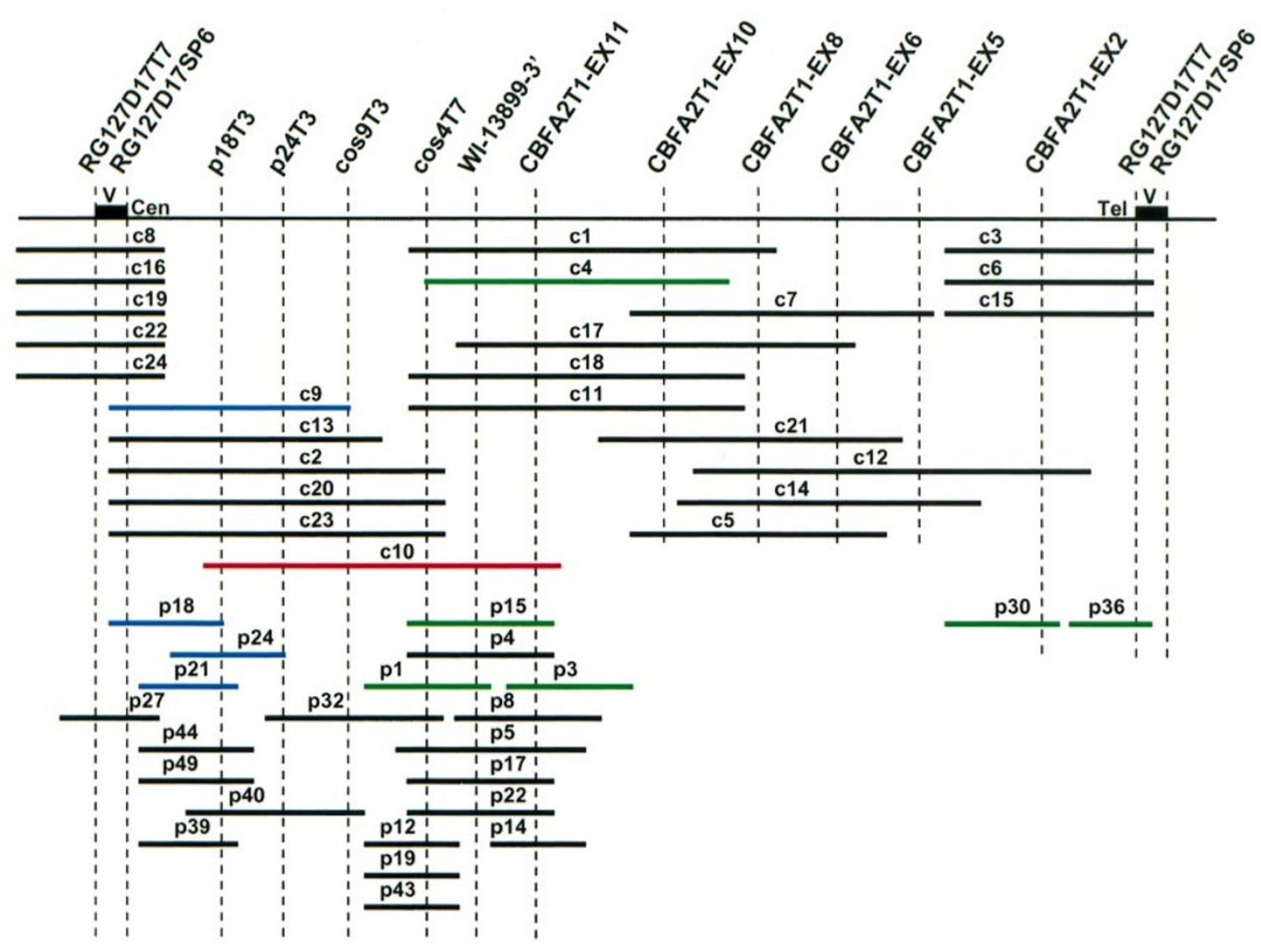

Figure 2 Cosmid and phage contig crossing the 8q22.1 breakpoint. Markers including STS and CBFA2T1 exons are indicated at the top. Clone numbers beginning with c indicate cosmid, p indicates phage. FISH experiments showed cosmid 9 and phages 18,21 and 24 (shown in blue) were mapped proximal to the breakpoint, cosmid 4 and phages 1, 3, 15, 30 and 36 (green) mapped distal, and cosmid 10 (red) spanned the breakpoint. V: BAC vector sequence, Cen: centromere, Tel: telomere. 


\section{$8 q 22.1$ region \\ Breakpoint

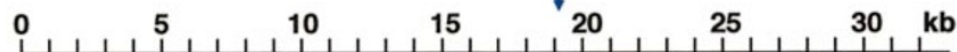

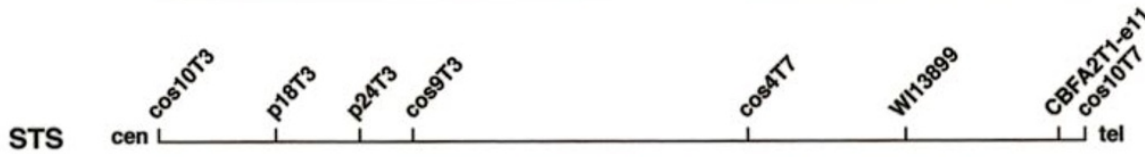

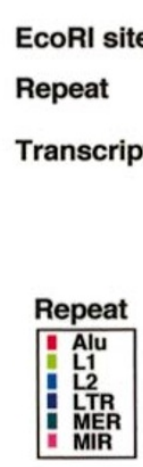

HERVH

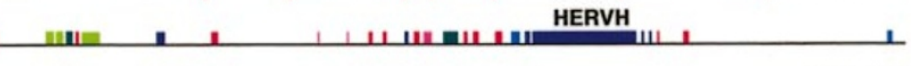

CBFA2T1-e11

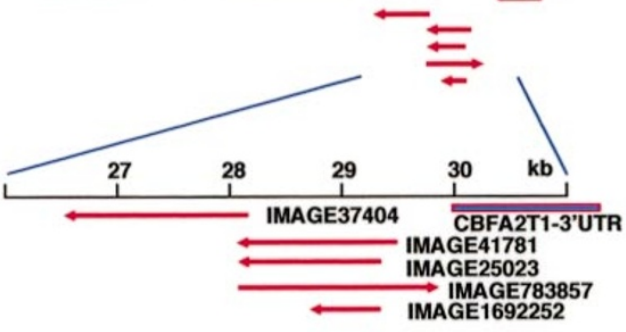

b

\section{1q21.1 region}
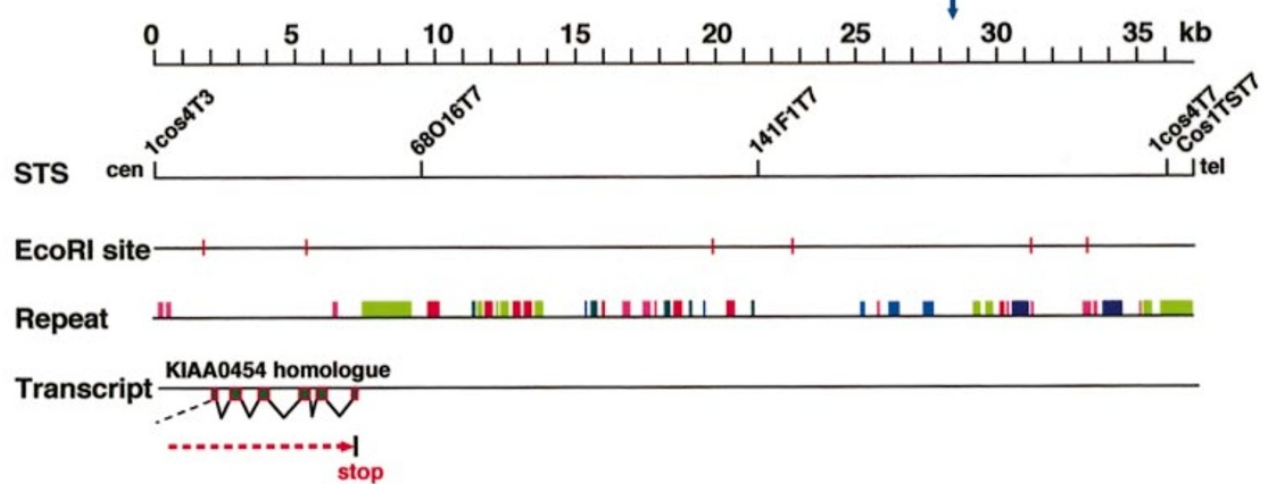

Figure 3 a Sequence characterization of cosmid 10 spanning the 8q22.1 breakpoint. Markers, EcoRl sites, repetitive sequences identified by RepeatMasker (http://ftp.genome.washington.edu/cgi-bin/RepeatMasker), and transcripts are indicated separately. The CBFA2T1 gene (exon 11) was $11 \mathrm{~kb}$ distal to the breakpoint b Sequence characterization of the 1cos4 spanning the 1q21.1 breakpoint. The same colors as in a were used to show repetitive sequences. A new gene homologous to KIAA0454 was found $21 \mathrm{~kb}$ proximal to the breakpoint.

TAG AAG AGG-3')/der(8)R (5'-AGC TIT ACA GCC ACT TCC CT-3') for the der(8) breakpoint were used. Breakpoint sequences of both $\operatorname{der}(1)$ and $\operatorname{der}(8)$ for all three individuals were identical and therefore appear to bestable in this family. BLAST2 comparison of cosmid 10 (8q22.1) and 1cos4 (1q21.1) sequences revealed several interspersed repetitive elements, such as Alu and L1 repeats, which were shared by both clones, but none of these repeats were close to the breakpoints. The two chromosomes did, however, show moderate similarity (62-69\%) in short unique sequences (26-29bp) immediately flanking the breakpoints (Figure4b). 


\section{a.}

\begin{tabular}{|c|c|}
\hline $\operatorname{ch} 8$ & AAGGATAAACATGGCTTCACTATCACCATCAGTG \\
\hline & 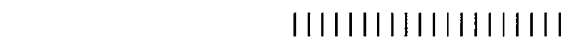 \\
\hline $\operatorname{der}(1)$ & CAGTTCATAAATACCTTCTTCTTCACTATCACCATCAGTG \\
\hline ch1 & $\begin{array}{l}|||||||||||||||||||| \\
\text { CAGTTCATAAATACCTTCTTTATGCTTTACATTTTTACTG }\end{array}$ \\
\hline $\operatorname{der}(8)$ & $\begin{array}{r}\|\|\|\|\|\|\|\|\|\|\|\| \| \\
\text { AAGGATAAACATGGCTICACTATGCTTTACATTTTTACTG }\end{array}$ \\
\hline & $\|1\||\||\||\||\||\||\||\|| \mid$ \\
\hline ch8 & AAGGATAAACATGGCTTCACTATCACCATCAGTG \\
\hline
\end{tabular}

b.

\begin{tabular}{|c|c|c|}
\hline \multirow[t]{2}{*}{ ch1 } & AAGCA-GTTCATAAATAC--CTTCTTTAT & $69 \%(18 / 26)$ \\
\hline & \|\|$\|111\| 111$ & \\
\hline ch8 & AAGCACAAGGATAAACATGGCTTCACTAT & $62 \%(18 / 29)$ \\
\hline
\end{tabular}

Figure 4 a Sequence comparison of normal chromosomes 1 and $8, \operatorname{der}(1)$, and $\operatorname{der}(8)$ at translocation breakpoints. Underlining indicates a duplication of six nucleotides from chromosome 8 b Short sequence (26-29bp) of similarity at breakpoints. Arrows mark chromosomal breakage sites.

\section{Mutation analysis of the CBFA2T1 gene in 37 GTS patients}

DNA from a total of 37 unrelated GTS patients was screened for mutations within all CBFA2T1 exons. The patients came from the Tourette Syndrome Association affected sib pair study ${ }^{16}$ and from the South African Afrikaner study. ${ }^{17}$ No nucleotide substitutions, deletions, or insertions were identified in the coding regions. One polymorphism, a T/C transition in intron 10, 35 bp upstream of exon 11, was found (nucleotide67 within GenBank sequence AF018282: AGTGTATTAA [T/C] TTGGGATCTT). However, the allele frequencies for this polymorphism were not significantly different in patients and controls. $T$ allele frequencies were $45 \%$ in patients and $50 \%$ in controls.

\section{Discussion}

We have characterized the translocation breakpoints from a GTS family with a balanced $(1 ; 8)(q 21.1 ; q 22.1)$ translocation. Although we did not find any genes that were directly disrupted by the breakpoints, we did identify the CBFA2T1 gene $11 \mathrm{~kb}$ distal to the $8 q 22.1$ breakpoint and a sequence closely related to the KIAA0454 gene $21 \mathrm{~kb}$ proximal to the 1q21.1 breakpoint. By sequencing the junction fragments, we also found that the translocation process involved the duplication of six nucleotides from chromosome 8.

The chromosome1 KIAA0454 gene homologue is an unlikely can didate for a GTS gene. KIAA0454 encodes a non- muscle myosin heavy chain A (NMMHC-A)-like protein which might play a role in smooth muscle cell proliferation. $^{30,31}$ The related 1q21.1 sequence showed $97 \%$ identity at a nucleotide level and $97 \%$ identity at an amino acid level with KIAA0454. However, the 1q21.1 sequence did not have any representative ESTs in dbEST as determined by BLASTN analysis. In addition, expression of this sequence was not observed in fetal brain cDNAs even though KIAA0454 expression was readily detected. The $1 q 21.1$ sequence related to KIAA0454 may therefore not be a functional gene. Other GTS gene mapping studies have also failed to implicate the 1q21.1 locus in this disorder.

In contrast, chromosome8q22.1 remains an attractive location for a GTS gene. Leppert and colleagues $^{23}$ obtained some of their best parametric lod scores at this site using a large, unilineal Utah GTS kindred. Simonic et $\mathrm{al}^{17}$ also found some of their most promising case/control association results using the isolated Afrikaner population of South Africa at this location. The Afrikaner results for $8 q 22.1$ have recently been confirmed by the Transmission Distortion Test (TDT) (un published results).

The CBFA2T1 gene remains a viable GTS candidate. CBFA2T 1 was originally identified through studies of chromosome8;21 translocations which frequently are found in acute myeloid leukemias. ${ }^{26,27}$ The hybrid protein resulting from the fusion of AML1 at 21q21.3 and CBFA2T 1 is believed to contribute to malignant transformation. The CBFA2T1 protein is a likely transcription factor because it has characteristic zinc finger DNA binding motifs and proline/serine/ threonine rich regions. ${ }^{32,33}$ CBFA2T1 is expressed at high levels in fetal and adult brain, heart, and skeletal muscle. CBFA2T 1 has a total of 13 exons spread over about $87 \mathrm{~kb}$ of genomic DNA. ${ }^{28}$ The nervy gene in Drosophila is similar to CBFA2T1 in sequence and is a target of the ultrabithorax homeotic gene product. ${ }^{34,35}$ CBFA2T1 coding, 5' and 3' sequences are very highly conserved between humans and mice. $^{36}$

Although CBFA2T1 was not directly disrupted by the translocation breakpoint in the GTS family we studied, it is still possible that the translocation significantly altered the expression of CBFA2T1 (or another nearby gene). Position effects of translocations have been reported for more than 10 human diseases (reviewed by Kleinjan et $\mathrm{al}^{37}$ ) Breakpoints have been found at both $5^{\prime}$ and $3^{\prime}$ sides of causative genes. Aniridia, Greig cephalopolysyndactyly, Saethre-Chotzen syndrome, sex reversal, facio-scapulo-humural muscular dystrophy, and split hand/split foot malformation have all been reported to be caused by translocations $3-450 \mathrm{~kb}$ downstream (3') of the genes. ${ }^{38-43}$

The sequences of the junction fragments for the translocation in the GTS family contribute to our knowledge of reciprocal translocations. Since the CBFA2T 1 breakpoints in leukemias usually occur between exons $1 \mathrm{la}$ and 2, the 8q22.1 breakpoint in the GTS family is completely different. Although Alu elements and other interspersed repeats are 
often involved in chromosomal rearrangements, ${ }^{44}$ no repeat sequences were found which could explain the translocation in the GTS family. The breakpoint regions of $1 q 21.1$ and 8q22.1 did, however, show moderate similarity in short sequences (26-29 bp) flanking the breakpoints which may have mediated chromosomal pairing.

Six nucleotides from chromosome8 but none from chromosome 1 were duplicated during generation of the translocation. The breakpoint sequences of several constitutional balanced translocations in human have now been determined. ${ }^{45-54}$ Most translocations appear to involve either deletion or duplication of at least a few bases at the breakpoints. Some translocations involve the insertion of bases unrelated to either of the parental chromosomes. The duplication of a few bases at the breakpoint is also reminiscent of transposition of Alu elements and other retroposons. ${ }^{44}$ It appears that translocations can occur by at least several quite different mechanisms. Compilation of additional examples will eventually improve our understanding.

Although the evidence is overwhelming that Gilles de la Tourette syndrome is highly heritable, it is also becoming clear that the genetics of GTS are much more complex than originally anticipated. A simple autosomal dominant mode of inheritance with reduced penetrance is no longer seriously considered. Candidate gene studies, parametric linkage mapping using large kindreds, an affected sib pair study and a genome-wide association study have all implicated quite different loci. Multiple genes as well as non-heritable factors almost certainly interact in complex ways to produce the GTS phenotype. Identifying the various GTS genes will be difficult. However, chromosomal rearrangements occurring in GTS patients like the chromosome 1;8 translocation studied here may facilitate identification of some of these genes.

\section{Acknowledgements}

We thank the Tourette Syndrome Association and especially Sue Levi-Pearl for alerting us to the translocation family and for many years of generous financial support. We also recognize Ms Julie A Kuc for expert assistance with the FISH experiments. GenBank accession numbers AF198490 and AF198494 apply.

\section{References}

1 Robertson MM, Stern JS: Gilles de la Tourette syndrome. Br J Hosp Med 1997; 58: 253-256.

2 Alsobrook JP II, Pauls DL: The genetics of Tourette syndrome. Neurol Clin 1997; 15: 381-393.

3 Barr CL, Sandor P: Current status of genetic studies of Gilles de la Tourette syndrome. Can J Psychiatry 1998; 43: 351-357.

4 Price RA, Kidd KK, Cohen DJ, Pauls DL, Leckman JF: A twin study of Tourette syndrome. Arch Gen Psychiatry 1985; 42: 815-820.

5 Hyde TM, Aaronson BA, Randolph C, Rickler KC, Weinberg DR: Relationship of birth weight to the phenotype expression of Gilles de la Tourette's syndrome in monozygotic twins. Neurology 1992; 42: 652-658.

6 Comings DE, Comings BG, Devor EJ, Cloninger CR: Detection of major gene for Gilles de la Tourette syndrome. Am J Hum Genet 1984; 36: 586-600.
7 Pauls DL, Leckman JF: The inheritance of Gilles de la Tourette syndrome and associated behaviors: evidence for autosomal dominant transmission. N Eng J Med 1986; 315: 993-997.

8 Eapen V, Pauls DL, Robertson MM: Evidence for autosomal dominant transmission in Tourette's syndrome: United Kingdom cohort study. Br J Psychiatry 1993; 162: 593-596.

9 Pakstis AJ, Heutink P, Pauls DL et al: Progress in the search for genetic linkage with Tourette syndrome: an exclusion map covering more than $50 \%$ of the autosomal genome. Am J Hum Genet 1991; 48: 281-294.

10 Wilkie PJ, Ahmann PA, Hardacre J, LaPlant RJ, Hiner BC, Weber JL: Application of microsatellite DNA polymorphisms to linkage mapping of Tourette syndrome gene(s). Adv Neurol 1992; 58: $173-180$.

11 van de Wetering BJM, Heutink P: The genetics of the Gilles de la Tourette syndrome: a review. J Lab Clin Med 1993; 121: 638-645.

12 Hasstedt SJ, Leppert M, Filloux F, van de Wetering BJM, M cMahon WM: Intermediate inheritance of Tourette syndrome, assuming assortative mating. Am J Hum Genet 1995; 57: 682-689.

13 Walkup JT, LaBuda MC, Singer HS, Brown J, Riddle MA, Hurko O: Family study and segregation analysis of Tourette syndrome: evidence for a mixed model of inheritance. Am J Hum Genet 1996; 59: 684-693.

14 Kurlan R, Eapen V, Stern J, McDermott MP, Robertson MM: Bilineal transmission in Tourette's syndrome families. Neurology 1994; 44: 2336-2342.

15 Lichter DG, Jackson LA, Schachter M: Clinical evidence of genomic imprinting in Tourette's syndrome. Neurology 1995; 45: 924-928.

16 The Tourette syndrome association international consortium for genetics: A complete genome screen in sib pairs affected by Gilles de la Tourette syndrome. Am J Hum Genet 1999; 65: 1428-1436.

17 Simonic I, Gericke GS, Ott J, Weber JL: Identification of genetic markers associated with Gilles de la Tourette syndrome in an Afrikaner population. Am J Hum Genet 1998; 63: 839-846.

18 Taylor LD, Krizman DB, Jankovich J et al: 9p monosomy in a patient with Gilles de la Tourette's syndrome. Neurology 1991; 41: 1513-1515.

19 Donnai D: Gene location in Tourette syndrome. Lancet 1987; 14: 627.

20 Boghosian-Sell L, Comings DE, Overhauser J: Tourette syndrome in a pedigree with a 7;18 translocation: identification of a YAC spanning the translocation breakpoint at 18q22.3. Am J Hum Genet 1996; 59: 999-1005.

21 Brett PM, Curtis D, Robertson MM, Dahlitz M, Gurling HM: Linkage analysis and exclusion of regions of chromosome 3 and 8 in Gilles de la Tourette syndrome following the identification of a balanced reciprocal translocation $46 \mathrm{XY}, \mathrm{t}(3 ; 8)(\mathrm{p} 21.3 ; \mathrm{q} 24.1)$ in a case of Tourette syndrome. Psychiatr Genet 1996; 6: 99-105.

22 Devor EJ, Magee HJ: Multiple childhood behavioral disorders (Tourette syndrome, multiple tics, ADHD, and OCD) presenting in a family with a balanced chromosome translocation $\mathrm{t}(1 ; 8)(\mathrm{q} 21.1 ; \mathrm{q} 22.1)$. Psychiatr Genet 1999; 9: 149-151.

23 Leppert M, Peiffer A, Snyder B et al: Two loci of interest in a family with Tourette syndrome. Am J Hum Genet 1996; 59(suppl): A225.

24 Chong SS, Pack SD, Roschke AV et al: A revision of the lissencephaly and Miller-Dieker syndrome critical regions in chromosome17p13.3. Hum Mol Genet 1997; 6: 147-155.

25 Matsumoto N, Pilz DT, Ledbetter DH: Genomic structure, chromosomal mapping, and expression pattern of human DCAMKL1 (KIAA0369), a homologue of DCX (XLIS). Genomics 1999; 56: 179-183.

26 Erickson P, Gao J, Chang K-S et al: Identification of breakpoint in $t(8 ; 21)$ acute myelogenous leukemia and isolation of a fusion transcript, AML1/ETO, with similarity to Drosophila segmentation gene, runt. Blood 1992; 80: 1825-1831. 
27 Miyoshi H, Kozu T, Shimizu K et al: The $\mathrm{t}(8 ; 21)$ translocation in acute myeloid leukemia results in production of an AML1-MTG8 fusion transcript. EMBO J 1993; 12: 2715-2721.

28 Wolford JK, Prochazka M: Structure and expression of the human MTG8/ETO gene. Gene 1998; 212: 103-109.

29 Seki N, Ohira M, Nagase T et al: Characterization of cDNA clones in size-fractionated cDNA libraries from human brain. DNA Res 1997; 4: 345-349.

30 Saez CG, Myers JC, Shows TB, Leinwand LA: Human nonmuscle myosin heavy chain mRNA: generation of diversity through alternative polyadenylation. Proc Natl Acad Sci USA 1990; 87: 1164-1168.

31 Simons $M$, Wang $M$, McBride OW et al: Human nonmuscle myosin heavy chains are encoded by two genes located on different chromosomes. Circ Res 1991; 69: 530-539.

32 Downing JR: The AML1-ETO chimaeric transcription factor in acute myeloid leukaemia. Br J Haematol 1999; 106: 296-308.

33 Erickson PF, Robinson M, Owens G, Drabkin HA: The ETO portion of acute myeloid leukemia $t(8 ; 21)$ fusion transcript encodes a highly evolutionarily conserved, putative transcription factor. Cancer Res 1994; 54: 1782-1786.

34 Feinstein PG, Kornfeld K, Hogness DS, Mann RS: Identification of homeotic target genes in Drosophila melanogaster including nervy, a proto-oncogene homologue. Genetics 1995; 140: 573-586.

35 Sacchi N, Tamanini F, Willemsen R, Denis-Donini S, Campiglio S, Hoogeveen AT: Subcellular localization of the oncoprotein MTG8 (CDR/ETO) in neural cells. Oncogene 1998; 16: 2609-2615.

36 Kitabayashi I, Ida K, Morohoshi F et al: The AML1-MTG8 leukemic fusion protein forms a complex with a novel member of the MTG8 (ETO/CDR) family, MTGR1. Mol Cell Biol 1998; 18: 846-858.

37 Kleinjan D-J, van Heyningen V: Position effect in human genetic disease. Hum Mol Genet 1998; 7: 1611-1618.

38 Fantes J, Redeker B, Breen $M$ et al: Aniridia-associated cytogenetic rearrangements suggest a position effect may cause the mutant phenotype. Hum Mol Genet 1995; 4: 415-422.

39 Vortkamp A, Gessler M, Grzeschik K-H: GLI3 zinc-finger gene interrupted by translocations in Greig syndrome families. Nature 1991; 352: 539-540.

40 Krebs I, Weis I, Hudler M et al: Translocation breakpoint maps 5 kb 3' from TWIST in a patient affected with Saethre-Chotzen syndrome. Hum Mol Genet 1997; 6: 1079-1086.

41 McElreavey K, Vilain E, Barbaux S et al: Loss of sequences 3 ' to the testis-determining gene, SRY, including the $Y$ pseudoautosomal boundary associated with partial testicular determination. Proc Natl Acad Sci USA 1996; 93: 8590-8594.
42 van Deutekom JCT, Lemmers RJLF, Grewal PK et al: Identification of the first gene (FRG1) from the FSHG region on human chromosome4q35. Hum Mol Genet 1996; 5: 581-590.

43 Crackower MA, Scherer SW, Rommens JM et al: Characterization of the split hand/split foot malformation locus SHFM1 at 7q21.3-q22.1 and analysis of a candidate gene for its expression during limb development. Hum Mol Genet 1996; 5: 571-579.

44 Deininger PL, Batzer MA: Alu repeats and human disease. Mol Genet Metab 1999; 67: 183-193.

45 Giacalone JP, Francke U: Common sequence motifs at the rearrangement sites of a constitutional/autosome translocation and associated deletion. Am J Hum Genet 1992; 50: 725-741.

46 Arai E, Ikeuchi T, Nakamura Y: Characterization of the translocation breakpoint on chromosome22q12.2 in a patient with neurofibromatosis type2 (NF2). Hum Mol Genet 1994; 3: 937-939.

47 van Bakel I, Holt S, Craig I, Boyd Y: Sequence analysis of the breakpoint regions of an $X ; 5$ translocation in a female with Duchenne muscular dystrophy. Am J Hum Genet 1995; 57: 329-336.

48 Holmes SE, Riazi MA, Gong W et al: Disruption of the clathrin heavy chain-like gene (CLTCL) associated with features of DGS/ VCFS: a balanced $(21 ; 22)(p 12 ; q 11)$. Hum Mol Genet 1997; 6: 357-367.

49 Kehrer-Sawatzki H, Haussler J, Krone W et al: The second case of a $t(17 ; 22)$ in a family with neurofibromatosis type 1 : sequence analysis of the breakpoint regions. Hum Genet 1997; 99: 237-247.

50 Ishikawa-Brush Y, Powell JF, Bolton P et al: Autism and multiple exostoses associated with an $\mathrm{X} ; 8$ translocation occurring within the GRPR gene and 3' to the SDC2 gene. Hum Mol Genet 1997; 6: 1241-1250.

51 Yoshiura K-I, Machida J, Daack-Hirsch S et al: Characterization of a novel gene disrupted by a balanced chromosomal translocation $\mathrm{t}(2 ; 19)(\mathrm{q} 11.2 ; \mathrm{q} 13.3)$ in a family with cleft lip and palate. Genomics 1998; 54: 231-240.

52 Kurahashi H, Sakamoto M, Ono J, Honda A, Okada S, Nakamura Y: Molecular cloning of the chromosomal breakpoint in the LISI gene of a patient with isolated lissencephaly and balanced $t(8 ; 17)$. Hum Genet 1998; 103: 189-192.

53 Ikegawa S, Masuno M, Kumano $\mathrm{Y}$ et al: Cloning of translocation breakpoints associated with Shwachman syndrome and identification of a candidate gene. Clin Genet 1999; 55: 466-472.

54 Holder Jr JL, Butte NF, Zinn AR: Profound obesity associated with a balanced translocation that disrupt the SIM 1 gene. Hum Mol Genet 2000; 9: 101-108. 